\title{
ON THE DISTRIBUTIONAL STIELTJES TRANSFORMATION
}

\author{
D. NIKOLIC - DESPOTOVIC and A. TAKACI \\ Institute of Mathematics \\ Novi Sad, Yugoslavia \\ (Received January 10, 1984 and in revised form June 24, 1985)
}

ABSTRACT. This paper is concerned with some general theorems on the distributional Stieltjes transformation. Some Abelian theorems are proved.

KEY WORISS AND PHRASES. Stiel,ties transforms of distributional, asymptotic behavior, Abf Zian theorems.

1.130 AMS SUBJECT CLASSIFICATION CODE. 44F12, $44 A 15$.

\section{REGULARLY VAPYIIJG FUNCTIONS}

Throughout the paper, $r$ will denote a positive continuous function on an interval $(x, \infty), x \geqslant 0$, sush that the limit

$$
\lim _{t \rightarrow \infty} \frac{r(p t)}{r(t)}
$$

exists for every $\mathrm{p}>0$. Sucin functions are called regularly varying fulıctions (r.v.f.) at infinity and it is well known ([7]) that they are of the form $r(t)=t^{a} L(t)$ for some a $\epsilon R$ (called the order or index of $r$ ) and some slowly varying function (s.v.f.) L. This means that the function $L:(X, \infty) \rightarrow(0, \infty)$ is continuous and that

$$
\lim _{t \rightarrow \infty} \frac{L(p t)}{L(t)}=1
$$

for every $\mathrm{p}>0$.

\section{QUASIASYMPTOTIC BEHAVIOUR AT INFINITY RELATED TO $r$}

The quasiasymptotic behaviour (q.a.b.) at infinity of tempered distributions with support in $[C, \infty)$ (denoted by $S_{+}^{\prime}$ ) $w \neq s$ defined by Zavijalov (see, for instance, [2]). In this paper we use a sornewhat inore general concept of $c, a, b$, related to a r.v.f. as defined and analysed in [10].

Definition 1. Let $\mathrm{T} \epsilon \mathrm{S}_{+}^{\prime}$ and $\mathrm{r}$ be some $\mathrm{r} \cdot \mathrm{v} . \mathrm{f}$. The distribution $\mathrm{T}$ has q.a.b. at infinity related to $r$ if there exists the limit in the sense of $\mathrm{S}^{-}$:

$$
\lim _{t \rightarrow \infty} \frac{T(k t)}{r(k)}=g(t)
$$

provided that $g \equiv 0$. 
If the order of $r$ is $a$, then $g(t)=A f_{a+1}(t)$ for some $A \neq 0$ (from now on we take $A=1$ for simplicity), where

$$
f_{a+1}(t)=H(t) t^{a} / \Gamma(a+1) \text { for } a \geqslant 0 \text { and } f_{a+1}(t)=D^{n} f_{a+n+1}(t)
$$

for $a<0$ and $n+a>0, n \in N$. As usual, $H$ is the characteristic function of the interval $(0, \infty)$, and $D$ stands for the distributional derivative.

It is easy to see that a continuous function on $(0, \infty)$ having ordinary asymptotic behaviour of order $a>-1$ related to $r$ has also q.a.b. of the same order and conversely. However for $a \leqslant-1$ this may not be true. This follows from the following

Structural Trieorem. ([10]) A distribution $\mathrm{T} \in \mathrm{S}_{+}^{\prime}$ has $\mathrm{q} \cdot \mathrm{a} \cdot \mathrm{b}$. at infinity related to a r.v.f. $r$ of order at iff there exist a natural number $n$, $\mathrm{n}+\mathrm{a}>\mathrm{C}$, and a continuous function $\mathrm{F}$ on $\mathrm{R}$ such that

$F=T * f_{n}$ and $F(t) \sim \frac{1}{\Gamma(n+a+1)} t^{n} r(t)$ as $t \rightarrow \infty$.

The proof of this important theorem is analogous to the one of Theorem I in [2], p. 373.

\section{EQUIVALENCE AT INFINITY}

The other "asymptotic behaviour" of distributions at infinity given in the following definition was used in [3], [1] and [6]; however, this notion goes back to Sebastiao e Silva ([8]).

Definition 2. A distribution $T \epsilon S_{+}^{-}$is equivalent at infinity to $r(t)=t^{a} L(t)$, a $z_{-}$, if for some $x^{-}, x^{-} \geqslant x$, and some nonnegative integer $\mathrm{n}, \mathrm{n}+\mathrm{a}>0$, there exists a continuous function $\mathrm{F}$ on $\left(\mathrm{X}^{-}, \infty\right)$ such that $\mathrm{T}=\mathrm{D}^{\mathrm{n}} \mathrm{F}$ on $\left(\mathrm{X}^{\top}, \infty\right)$ and

$$
F(t) \sim t^{n} r(t) /(a+1)(a+2) \ldots(a+n)
$$

in the ordinary sense as $t \rightarrow \infty$.

It seems to be of interest to compare these two asymptotics; for our purposes it is enough to prove

Lemma 1. Let $T \epsilon S_{+}^{\prime}$ be equivalent at infinity to $r(t)=t^{a} L(t)$ for $a>-1$. Then it has q.a.b. of order a related to $r$.

Proof. We can write $T=B+D^{n} F(t)$, where the supports of $B$ and $F$ are, respectively, in $\left[0, X^{\prime}\right]$ and $\left[X^{-}, \infty\right), X^{-} \geqslant 1$. Let us prove that

$$
\lim _{k \rightarrow \infty} \frac{B(k t)}{k^{a} L(k)}=0
$$

In fact, for every $\varepsilon>0$ there exists a number $n_{1} \epsilon N_{0}\left(N_{0}=N U\{0\}\right)$ and a continuous function $F_{1}$ on $R$ such that $D^{n_{1}} F_{1}=B$ and $\operatorname{supp} F_{1} c[-\varepsilon$, $\left.X^{\top}+\varepsilon\right]$. For $\phi \epsilon$ we have

$$
\begin{aligned}
& <\frac{B(k t)}{k^{a} L(k)}, \phi(t)>=\left\langle\frac{F_{1}(t)}{k^{n_{1}+a+1} L(k)},(-1)^{n_{1}}{ }^{\left(n_{1}\right)}(t / k)>=\right. \\
& =\frac{(-1)^{n_{1}}}{k^{n_{1}+a+1} L(k)} \int_{-\varepsilon}^{X^{\prime}+\varepsilon} F_{1}(t) \phi^{\left(n_{1}\right)}(t / k) d t \rightarrow 0
\end{aligned}
$$


since $n_{1}+a+1 \geqslant a+1>0$. By supposition $F$ satisfies (3.1), so by the Structural theorem it has $q . a . b$. of order a related to $r$.

4. STIELTJES TRANSFORM OF DISTRIBUTIONS

For the sake of completness we rewrite the definition of the distributional Stieltjes transform given in [4]. Let $I^{\prime}(z), z \epsilon$, denote the subspace of distributions $t \epsilon S_{+}^{-}$such that $T=D^{n} G$ for some $n \epsilon N$ and some locally integrable function $G$ on $R$ with support in $[0, \infty)$ and

$$
\int^{\infty}|G(t)| t^{-(z+n+1)} d t<\infty \text {. }
$$

From now on we take $z \epsilon R$ and $z>-1$, though a complex setting is also possible (see [4] or [1]). Obviously $I^{\prime}(z) c_{+}^{\prime}$ and $I^{\prime}\left(z_{1}\right) c^{\prime}\left(z_{2}\right)$ for $-1<z_{1}<z_{2}$

Lefinition 3. The stieitjes transf $\mathrm{rm}$ of inlex $\mathrm{z}$ of a distribution T $\epsilon I^{\prime}(z)$ is the complex valued function

$$
\mathrm{S}_{z}\{\mathrm{~T}\}(s)=\left\langle\mathrm{T}(\mathrm{t}), \frac{\mathrm{h}(\mathrm{t})}{(\mathrm{t}+\mathrm{s})^{z+1}}\right\rangle, \quad s \in \mathrm{c} \backslash(-\infty, 0], \quad \text {, }
$$

where $h$ is an infinitely differentiable function on $R$ such that $h(t)=1$ in sume neignbourhood of $[0, \infty)$ and $h(t)=0$ in some interval $(-\infty,-\varepsilon)$, $\varepsilon>0$.

It is easy to see that (4.1) does not depend on the function $h$, so it is usually omitted. It is proved in [5] that $S_{z}\{T\}(s)$ is a holonorphic function of the complex variable $s$ in the domain $C \backslash(-\infty, 0)$ provided that $T_{\epsilon} I^{\prime}(z)$. We shall need the following equality ([5], p. 140)

$$
S_{z+n}\{T\}(s)=\frac{1}{(z+1)(z+2) \ldots(z+n)} S_{z}\left\{D^{n} T\right\}(s)
$$

for $\mathrm{T} \epsilon \mathrm{I}^{\prime}(\mathrm{z})$ and $\mathrm{n} \epsilon \mathrm{N}$. Observe that $\mathrm{T} \epsilon \mathrm{I}^{\prime}(z)$ implies $\mathrm{T} \epsilon \mathrm{I}^{\prime}(z+n)$ and $D^{n} \in I^{\prime}(z)$.

\section{5: ABELIAN THEOREMS}

The initial value type Abelian theorems for the distributional Stieltjes transform seem to have a satisfactory form. So, we prove only final value type ones. We use first the following result from [6]:

Theorem 1. Let us suppose that $T_{\epsilon} I^{\prime}(z)$ is equivalent at infinity to a regularly varying function $r(t)=t^{a} L(t)$ of order $a>-1$. Then

$$
S_{z}\{T\}(s) \sim B(a+1, z-a) L(s) s^{a-z} \text { as } s \rightarrow \infty, s \epsilon R,
$$

provided that $\mathrm{z}>\mathrm{a}>-1$.

As usual, $B(p, q)$ stands for the beta function. In view of Lemina 1 we see that this Theorem can be rewritten as

Theorem 1'. Let us suppose that $T \epsilon s_{+}^{\prime}$ has $q \cdot a \cdot b$. of order $a>-1$ related to the r.v.f. $r(t)=t^{a} L(t)$. Then $(5.1)$ holds if $z>a>-1$.

If $T$ in these two theorems is a continuous function on $(0, \infty)$, then $T(t) \sim t^{a} L(t)$ as $t \rightarrow \infty$ in the ordinary sense. Essentially, we need such 
a "functional" (i.e. rot "distributional") version of them in the following

Abelian Theorem. Let $\mathrm{T} \epsilon \mathrm{S}_{+}^{\prime}$ have $c_{\mathbf{t}}$.a.b. of order a related to a r.v.f. $r(t)=t^{a} L(t)$. Then

i) $\quad \mathrm{T} \in \mathrm{I}^{\prime}(\mathrm{z})$ for $z>\max (-1, \mathrm{a})$

and

ii) $S_{z}\{T\}(s) \sim \frac{\Gamma(z-a)}{\Gamma(z+1)} L(s) s^{a-z}$ as $s \rightarrow \infty$, staying on the real line.

Remark. Such a statement was proved in [3] for $r(t)=t^{z}$, $a>-1$ and ir [4] for $r(t)=t^{a} \log ^{j} t, a>-1$. Further on, r.v.f. were used ir: [6] (again for $a>-1$ ). In all these papers the equivalence at infinity was used. The q.a.b. was used in [9] for $r(t)=a^{a}$ (a - arbitrary real number) and now for any r.v.f. . In [1] the results from [3] ere gjven in a complex setting; it night be of interest to prove an analogous statement for our Abeliart theorem.

Proo: cf the kbeitian thsorem. Pant i) follows from the structural theorem and the estimate $L(t) \leqslant C_{\varepsilon} t^{\varepsilon}$ for $t \geqslant t_{0}=t_{0}(\varepsilon)(\varepsilon$ in $(0,1))$. For $i \dot{i}$, we take $n>-a$ and $F$ as in the structural theorem; then

$$
F(t) \sim C_{n} t^{n+a} L(t) \text { as } t \rightarrow \infty
$$

for $C_{n}=1 / \Gamma(n+a+1)$, By' Theorem $1^{\prime}$ we get

$$
S_{z+n}\{F\}(s) \sim C_{n} B(n+a+1, z+n-(n+a)) L(s) s^{a-z} \text { as } s \rightarrow \infty,
$$

and from $(4.2)$ we have

$$
\begin{aligned}
& S_{z}\{T\}(s) \sim(z+1)(z+2) \ldots(z+n) S_{z+n}\{F\}(s) \text {, so } \\
& S_{z}\{T\}(s) \sim c_{n} \frac{\Gamma(n+a+1) \Gamma(z-a)}{\Gamma(z+1)} L(s) s^{a-z} .
\end{aligned}
$$

This gives the statement $i i)$.

Example. The equivalence at infinity with the distribution

$$
T=A(a, j) F p\left(t^{a} \log _{+}^{j} t\right), \quad a \epsilon R, j \in N_{0}
$$

for appropriate constant $A(a, j)$ was analysed in [4]: Fp stands for the finite part. Obviously, $T$ is equivalent at infinity to $t^{a} \log ^{j} t$ for $a \notin Z_{-}$; we take $A(a, j)=1$ then. On the other hand, $T$ has q.a.b. of order a related to $t^{a} \log ^{j} t$ for $a \notin Z$ - and related to $A(a, j) t^{a} \log ^{j+l_{t}}$ for $a \in Z_{-}$; we take $A(a, j)=(-1)^{-a-1 /((-a-1) !(j+1))}$ then. Computing the Stieltjes transform of $T$ we see that it behaves at infinity as the Abelian theorem predicts (see [4], formulae (2.3) and (2.4)).

Now let $-2<a<-1$. Then the distribution $S=T+\delta$ has $q \cdot a \cdot b$. of order -1 related to $1 / t$ and is equivalent at infinity with $t^{2} \log ^{j}$. But for $z>-1$

$$
S_{z}\{S\}=S_{z}\{T\}+S_{z}\{\delta\} \sim C_{a, j} \log ^{j+1} s s^{a-z}+s^{-(z+1)} \sim s^{-(z+1)}
$$

when $s \rightarrow \infty$. This trivial example (which can be generalized easily) shows 
again that q.a.b. is more appropriate for final value type Abelian theorems for stieltjes transformation than equivalence at infinity, though the latter seems more "natural".

\section{REFERENCES}

1. CARMICHAEL, R.D. and MILTON, E.O. Abelian theorems for the distributional Stieltjes transform, J. Math. Anal. Appl., Vol. 72, No. 1, (1979), $195-205$.

2. DROZZZZINOV, JU.N. and ZAVIJALOV, B.I. Quasiasymptotic behaviour of generalized functions and Tauberian theorems in complex domain (in Russian), Mat. Sbornik, Tom 102 (144), No. 3, (1977), 372 - 390 .

3. LAVOINE, J. and MISRA, O.P. Théorémes abeliens pour la transformation de Stieltjes des distributions, (.R. Acad. Sci. Paris $279(1974), 99-102$.

4. LAVOIINE, I. and MTSRA, O.P. Abelian theorems for the distributional Stieltjes transformation, Math. Proc. Phil. Soc. 86 (1979), $287-293$.

5. LAVOINE, J. ard MISRA, O.F. Sur la transformation de Stieltjes ces distributions et son inversion au moyen de la transforstation de Laplace, C.R. Acad. Sci. Paris, 290 (1980), $139-142$.

6. MARIE, V., SKENDŽIC, M. and TAKACI, A. On Stieltjes transform of distributions behaving as regularly varying functions, (to af)pear).

7. SENETA, E. Reglilarly Varying functions, Springer Verlag Berlin, Heidelberg, New York, Lecture Notes in Math. 508 (1976).

3. SILVA, ¿.S.e. Integrals and order of growth of distributions, Inst. Cubelkijan de Ciencia, Lisboa 35 (1964), 7124.

3. TAKACI, A. A note on the distributional stieltjes transformation, Math. Proc. Phil. Soc., 94 (1983), 523 - 527.

10. VLADIMIROV, V.S. and ZAVIJALOV, B.I. Tauberian theoremis in the quantum field theory, (in Russian), Itogi Nauki i Tehniki, Serija Sovremennie problemi matematiki, Tor 15 (1980), Moskva. 


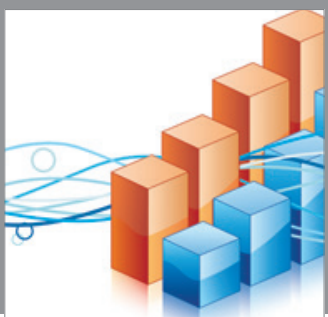

Advances in

Operations Research

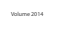

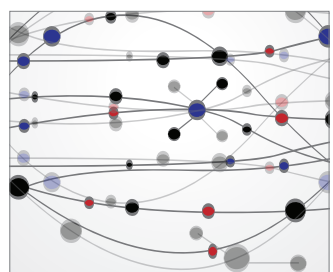

\section{The Scientific} World Journal
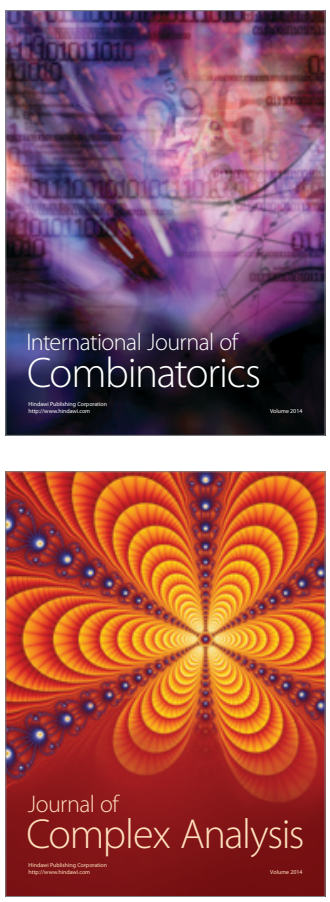

International Journal of

Mathematics and

Mathematical

Sciences
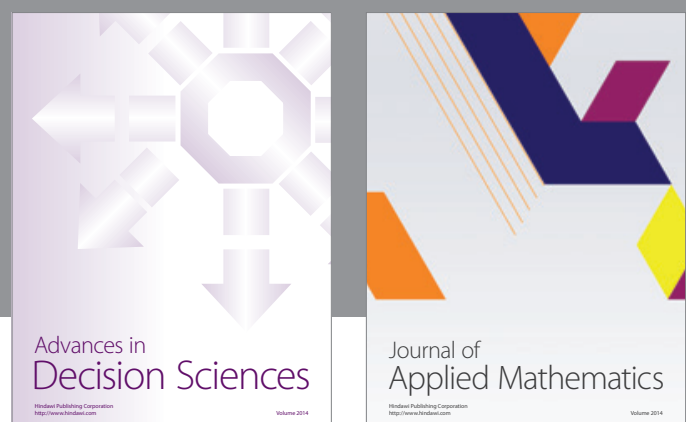

Journal of

Applied Mathematics
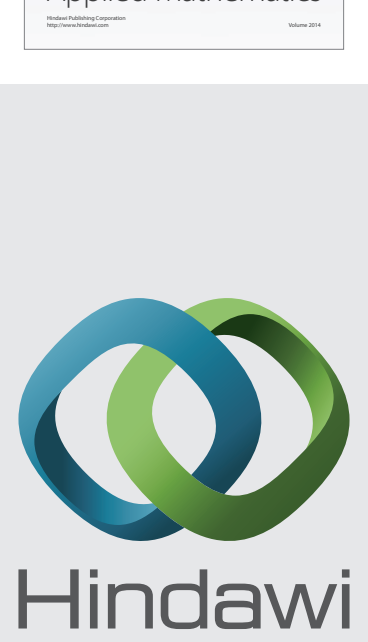

Submit your manuscripts at http://www.hindawi.com
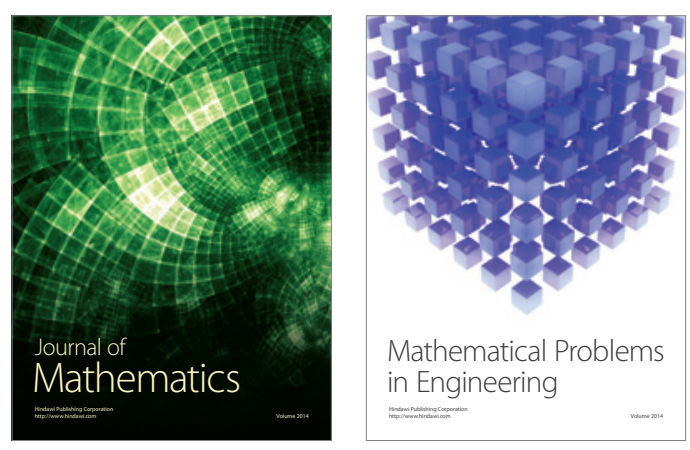

Mathematical Problems in Engineering
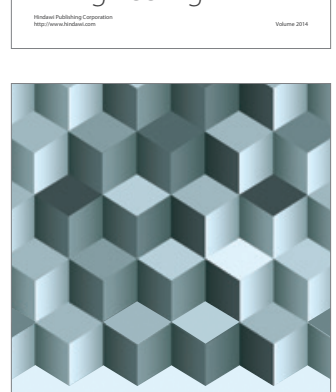

Journal of

Function Spaces
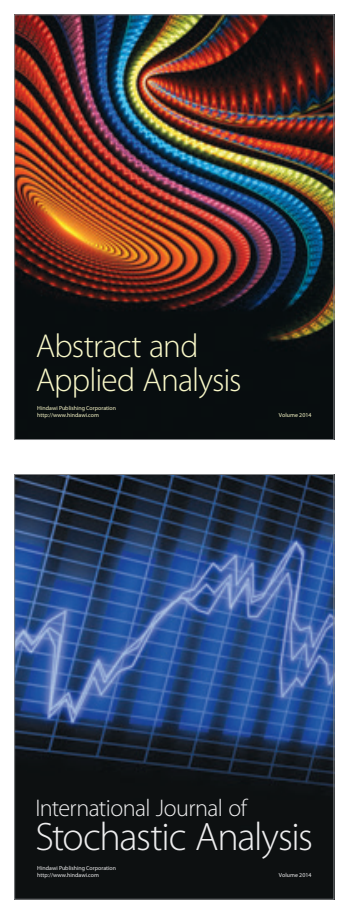

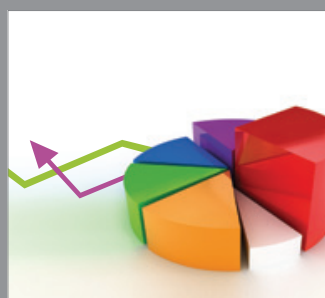

ournal of

Probability and Statistics

Promensencen
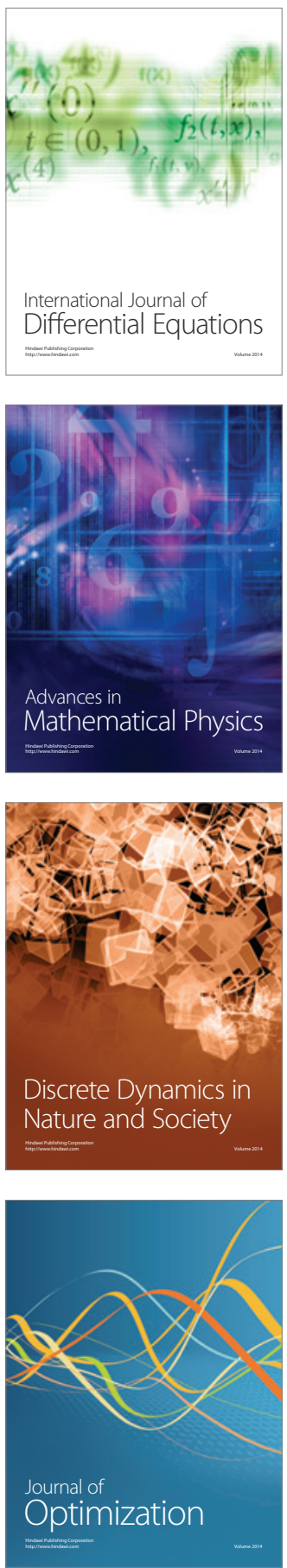